\title{
Diet-induced Atherosclerosis Increases the Release of Nitrogen Oxides from Rabbit Aorta
}

Robert L. Minor, Jr., Paul R. Myers, Ricardo Guerra, Jr., James N. Bates, and David G. Harrison Departments of Internal Medicine and Anesthesiology, and the Cardiovascular Center, University of Iowa College of Medicine, and Veterans Administration Medical Center, Iowa City, Iowa 52242

\section{Abstract}

We examined the hypothesis that impaired endothelium-dependent vasodilatation in atherosclerosis is associated with decreased synthesis of nitrogen oxides by the vascular endothelium. The descending thoracic aortae of rabbits fed either normal diet, a high cholesterol diet for 2-5 wk (hypercholesterolemic, HC), or a high cholesterol diet for 6 mo (atherosclerotic, AS) were perfused in a bioassay organ chamber with physiologic buffer containing indomethacin. Despite a dramatic impairment in the vasodilator activity of endotheliumdependent relaxing factor (EDRF) released from both $\mathrm{HC}$ and AS aortae (assessed by bioassay), the release of nitrogen oxides (measured by chemiluminescence) from these vessels was not reduced, but markedly increased compared to NL. Thus, impaired endothelium-dependent relaxation in atherosclerosis is neither due to decreased activity of the enzyme responsible for the production of nitrogen oxides from arginine nor to arginine deficiency. Because the production of nitrogen oxides increased in response to acetylcholine in both hypercholesterolemic and atherosclerotic vessels, impairments in signal transduction are not responsible for abnormal endothelium-dependent relaxations. Impaired vasodilator activity of EDRF by cholesterol feeding may result from loss of incorporation of nitric oxide into a more potent parent compound, or accelerated degradation of EDRF. (J. Clin. Invest. 1990. 86:2109-2116.) Key words: hypercholesterolemia • atherosclerosis • EDRF • nitric oxide $\bullet$ nitrosylated compounds $\bullet$ bioassay - chemiluminescence

\section{Introduction}

The endothelium-derived relaxing factor (EDRF) ${ }^{1}$ is a labile non-prostanoid compound with potent vasodilator properties, which is either nitric oxide or a related nitrosylated compound $(1,4)$. An enzyme-responsible nitric oxide production by oxidation of the guanidino nitrogens of arginine, recently termed nitric oxide synthetase, has now been identified in several

Address correspondence and reprint requests to Dr. David G. Harrison, Drawer LL, Cardiology Division, Emory University School of Medicine, Atlanta, GA 30322. Dr. Myers' present address is Dalton Research Center, The University of Missouri, Columbia, MO 63211.

Received for publication 30 January 1990 and in revised form 26 June 1990

1. Abbreviations used in this paper: EDRF, endothelium-derived relaxing factor; L-NMMA, L-N $N^{\mathrm{G}}$-monomethyl-arginine.

J. Clin. Invest.

(C) The American Society for Clinical Investigation, Inc.

0021-9738/90/12/2109/08 \$2.00

Volume 86, December 1990, 2109-2116 mammalian tissues including brain $(5,6)$, hepatocytes $(7)$, lung (8), macrophages $(9,10)$, and endothelial cells (11-14). This enzyme requires NADPH $(5,8,14)$, calmodulin $(15)$, and the macrophage enzyme has been reported to require biopterin (16). Recently, a $150-\mathrm{kD}$ protein with the properties of this enzyme had been purified to homogeneity from brain homogenate (15). It has also been suggested that endothelial cells possess both a calcium-dependent enzyme located in the cytoplasm and a membrane-associated, calcium-independent enzyme that participate in the synthesis of nitric oxide (13).

Atherosclerosis impairs endothelium-dependent vasodilation in both animal models (17-23) and human coronary arteries $(24,25)$, and thereby may predispose to vasoconstriction and arterial spasm. Bioassay studies have suggested that the mechanism underlying this defect in endothelial cell function is decreased EDRF production or release of a defective EDRF (26). It is therefore reasonable to suspect that cholesterol feeding and atherosclerosis might impair the function of the endothelial cell nitric oxide synthetase, either by a direct effect on the enzyme or by depletion of one or more of the required co-factors. Alternatively, it has been suggested that cholesterol feeding may alter endothelial cell receptors or second messenger systems such that the enzyme is not normally activated upon agonist stimulation (24).

In these experiments, we sought to examine the effect of both short- and long-term cholesterol feeding on the enzymatic process leading to the production of nitrogen oxides by the vascular endothelium. We employed a chemiluminescence technique to allow accurate determination of the release of nitric oxide and one electron oxidation product of nitric oxide from rabbit aorta under basal conditions and during administration of either acetylcholine or the calcium ionophore. The vasorelaxant activity of EDRF released from these vessels was concomitantly quantified by bioassay.

\section{Methods}

Cholesterol feeding protocol. New Zealand white rabbits were randomly assigned to treatment and control groups. Hypercholesterolemia was induced by feeding a $1 \%$ cholesterol diet for 2-5 wk $(n=7)$. Atherosclerosis was caused by continuing the $1 \%$ diet for $4 \mathrm{mo}$, followed by a $0.5 \%$ diet for $2 \mathrm{mo}(n=8)$. Age-matched controls $(n=10)$ were maintained on standard rabbit chow (Continental Grain, Chicago, IL).

Preparation of bioassay vascular ring for detection of EDRF. Bioassay detector rings were prepared from 3-5-mm segments of the proximal left circumflex coronary arteries of pigs. The endothelium was denuded by gently rubbing the intimal surface with the closed tip of forceps. The bioassay arterial ring was then mounted on two wire stirrups, one of which was immobile and the other connected to a force transducer (FTO3C; Gould, Cleveland, $\mathrm{OH}$ ) for recording isometric tension development. The rings were superfused by a physiologic buffer solution (Kreb's buffer of the following millimolar composition: $118.3 \mathrm{NaCl}, 4.7 \mathrm{KCl}, 1.88 \mathrm{CaCl}_{2}, 1.2 \mathrm{MgSO}_{4}, 1.2 \mathrm{KH}_{2} \mathrm{PO}_{4}, 25$ 
$\mathrm{NaHCO}_{3}, 11.1$ glucose, pH 7.40, warmed to $37^{\circ} \mathrm{C}$, and aerated with $95 \% \mathrm{O}_{2},\left(5 \% \mathrm{CO}_{2}\right)$, or by effluent from an aortic segment suspended in an organ bath (Fig. 1). Rates of superfusion were identical ( $4 \mathrm{ml} / \mathrm{min}$ ).

Before each study, the detector rings were gradually stretched to an optimal resting tension of $1-2 \mathrm{~g}$ over a period of $2 \mathrm{~h}$. Active tension of 3-4 $\mathrm{g}$ was then obtained by administration of $0.1-1 \mu \mathrm{M}$ of prostaglandin $F_{2 \alpha}$. Denudation was confirmed by the absence of relaxation to direct application of bradykinin $(0.01 \mu \mathrm{M})$.

Preparation of thoracic rabbit aorta as EDRF donor. On the day of the study, rabbits were killed by an overdose of pentobarbital. The descending thoracic aorta was isolated and removed, taking care to not damage the endothelium. The segment was immediately cannulated with stainless steel cannulas and mounted in an organ bath filled with Kreb's solution. An identical solution was continuously perfused in the direction of normal flow through the aortic segment. The aortic effluent could be directed to either the bioassay vascular segment, or to the collection chamber of the nitric oxide analyzer (Fig. 1).

Measurement of nitric oxide by chemiluminescence. The effluent from the aortic segment was directed into a collection chamber containing a solution of $1 \% \mathrm{NaI}$ and glacial acetic acid under reflux conditions (Fig. 1). This strong reducing environment is required for preserving nitric oxide in its authentic form, and also converts a number of nitrosyl compounds (including secondary nitrosoamines, nitrosothiols, and nitrite) to nitric oxide (4). A constant stream of inert nitrogen gas under vacuum carried nitric oxide gas released from this chamber into the nitric oxide analyzer (Dasibi, model 2108; Glendale, CA) where the gas was exposed to ozone at $45^{\circ} \mathrm{C}$. The reaction of ozone with nitric oxide releases light at a wavelength of 6,500-8,000 $\AA$. The amount of light generated by this reaction is linearly related to the quantity of nitric oxide present. The emitted light was detected by a photomultiplier tube, generating a DC signal, which was recorded on an oscillographic direct writing recorder.

Drugs. Bradykinin, acetylcholine, atropine, the calcium ionophore A23187, and indomethacin were all obtained from Sigma Chemical Co. (St. Louis, MO). L- $N^{G}$-mono-methyl-arginine (L-NMMA) was obtained from Calbiochem-Behring Corp. (La Jolla, CA). Prostaglandin $F_{2 \alpha}$ was obtained from Upjohn (Chicago, IL). Drugs were dissolved in distilled water and subsequently diluted in the physiologic buffer to desired concentrations, except for indomethacin, which was dissolved in $0.9 \% \mathrm{NaCl}$ with the $\mathrm{pH}$ adjusted to 8 by addition of $1 \mathrm{~N} \mathrm{NaOH}$. All agents were prepared immediately before experiments.

Protocol. All studies were performed in the presence of indomethacin $(1 \mu \mathrm{M})$ to inhibit the synthesis of cyclooxygenase products. Measurements of EDRF and nitrogen oxides (NO, $\mathrm{NO}_{2}^{-}, \mathrm{R}-\mathrm{NO}$ ) within the effluent of aortic segments were made after 10 min of perfusion with control physiologic buffer. Transit times from the aortic segment to the detector vessel and to the reflux chamber of the nitric oxide analyzer were identical $(3 \mathrm{~s})$. The physiologic buffer was infused at the rate of 4 $\mathrm{ml} / \mathrm{min}$ through both the direct superfusion channel and the aortic segments.

The bioassay detector vessel was initially superfused through the direct channel, bypassing the donor aortic segment. After a stable preconstriction was obtained, effluent from the rabbit aorta was directed onto the detector vessel, and the basal response was observed (Fig. 2). When the response reached a stable baseline, the effluent was directed into the collection chamber of the nitric oxide analyzer, where it underwent reduction preprocessing. Nitric oxide that was recovered from the effluent was then measured by chemiluminescence.

The release of EDRF and nitrosyl compounds in response to acetylcholine $(1 \mu \mathrm{M})$ was then examined. During administration of acetylcholine, atropine $(10 \mu \mathrm{M})$ was simultaneously infused onto the detector vessel to block smooth muscle muscarinic receptors. Acetylcholine was infused at a constant rate into the aortic segment, while effluent from the aorta remained directed to the denuded bioassay detector vessel. After a stable relaxation was recorded, the effluent was directed into the collection chamber of the nitric oxide analyzer and nitric oxide measurements were recorded.

An identical protocol was subsequently employed to examine the effect of the calcium ionophore A23187 $(10 \mu \mathrm{M})$. Before the administration of A23187, a second basal response was recorded. An interval of at least $15 \mathrm{~min}$ was allowed after cessation of acetylcholine before infusions of A23187.

In studies where L-NMMA was employed, this compound was infused proximal to the aortic segments at least $10 \mathrm{~min}$ before examining basal and agonist-stimulated production of EDRF and nitric oxide.

Cholesterol determinations. Blood samples were obtained directly from the heart at the time of death, and total serum cholesterol levels were determined as previously described (27).

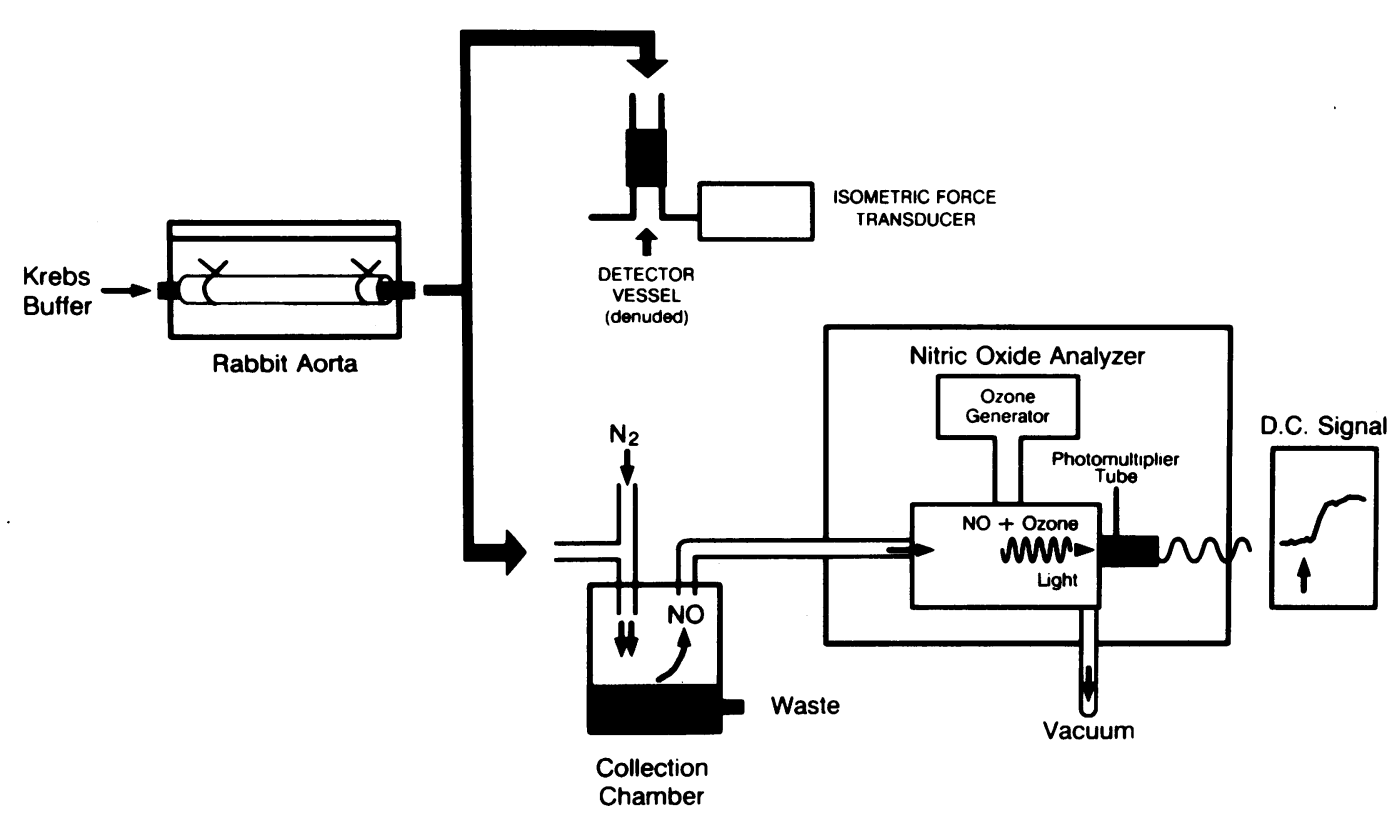

Figure 1. Experimental preparation used to quantify EDRF and nitric oxide release. Effluent from aortic segments first superfused a detector vessel for recording of bioassay responses. At the point of maximal response, effluent was immediately diverted into the collection chamber containing $1 \%$ sodium iodide in glacial acetic acid heated to a slow boil. After reduction preprocessing, recovered nitric oxide was transported in a stream of nitrogen under negative pressure into the nitric oxide analyzer and exposed to ozone. Light emitted by the resultant chemilumi-

nescence reaction was detected by a photomultiplier tube, generating a DC signal proportional to the amount of nitric oxide present. Transit times for vessel effluent to the detector vessel and the collection chamber were identical $(3,5)$. 

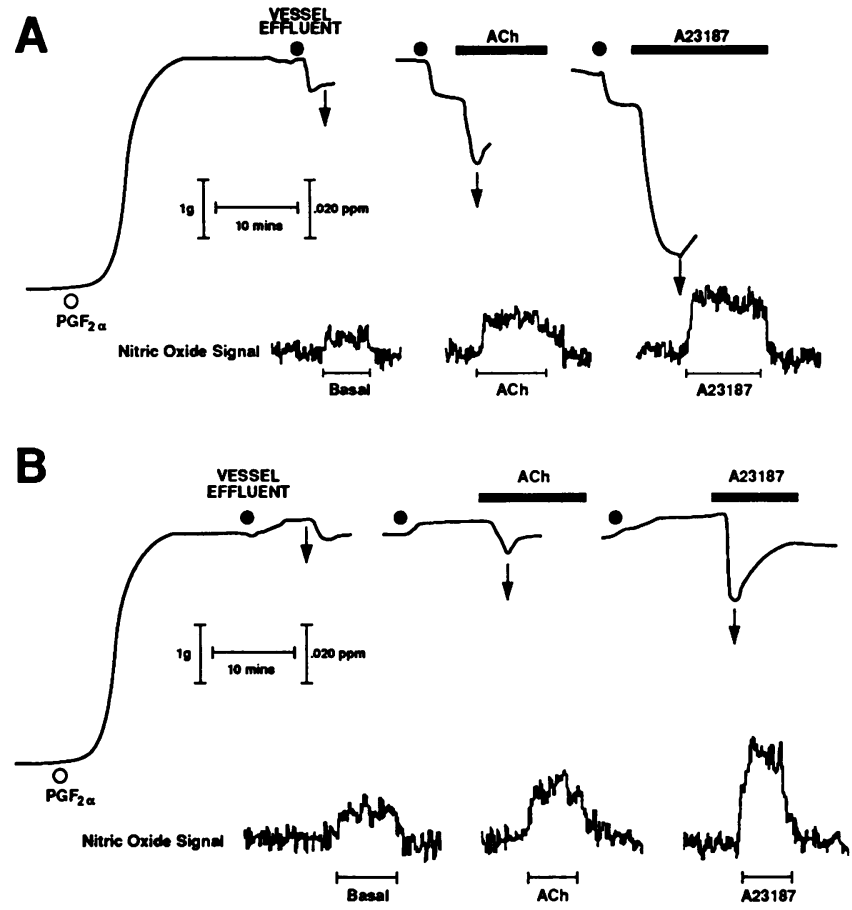

Figure 2. Representative experimental recordings from normal $(A)$ and atherosclerotic $(B)$ rabbit aorta. Control solution was initially superfused onto the detector vessel and preconstricted tension was obtained. Effluent from the aortic segment was then directed to the ring detector (๑) and basal responses were recorded. Effluent was then immediately diverted into the reflux chamber of the nitric oxide analyzer ( $\downarrow$ ), and nitric oxide signals were recorded, as control solution was again superfused onto the detector. This protocol was repeated during administration of acetylcholine $(1 \mu \mathrm{M})$ and subsequently the calcium ionophore $(10 \mu \mathrm{M})$. During administration of Ach, atropine $(10 \mu \mathrm{M})$ was directly superfused onto the detector.

Histology and electron microscopy studies of aortic segments and endothelium. $1 \mathrm{~cm}$-segments of the upper thoracic aorta were fixed in $10 \%$ formaldehyde, and sectioned for microscopic evaluation of intimal lesions and endothelial integrity. In addition, an adjacent segment was fixed in 3\% glutaraldehyde and processed for scanning and transmission electron microscopy.

Data analysis. Data are presented as mean \pm SEM. Responses of the detector ring to vasoactive substances released under basal conditions were expressed as a percentage of preconstricted tension. In the case of the hypercholesterolemic and atherosclerotic vessels, the effluent caused a slight paradoxic vasoconstriction, expressed as a negative value. Traditionally, relaxations of vascular rings are expressed in a cumulative fashion, i.e., the relaxation to acetylcholine and the relaxation due to basally released EDRF added together and expressed as a percent of the preconstricted tension. As effluent from hypercholesterolemic and atherosclerotic vessels produced a slight vasoconstriction under basal conditions, responses to either acetylcholine or A23187 were expressed using two approaches. The traditional (cumulative) approach used the formula: $\%$ relaxation $=$ (grams tension before basal response - grams tension after relaxation/grams tension before basal response).

In addition we analyzed relaxations to acetylcholine and A23187 excluding the basal response. This analysis employed the formula: \% relaxation $=$ (grams tension after basal response - grams remaining after relaxation/grams tension after basal response).

The amount of nitric oxide recovered from the aortic effluent was expressed as parts per million. Data regarding EDRF vasodilator activity (bioassay) and nitric oxide release (chemiluminescence) were iden- tical for both short-term and long-term age-matched control groups, and therefore combined for statistical analyses. Statistical significance was assessed by analysis of variance. When differences were indicated, a Scheffe's test was used for comparison between groups. Differences were considered significant when $P<0.05$.

\section{Results}

Plasma cholesterol levels. The plasma cholesterol levels were $38 \pm 4,1448 \pm 62$, and $1768 \pm 244 \mathrm{mg} / \mathrm{dl}$ for the control, shortterm, and long-term cholesterol fed groups, respectively.

Morphologic changes. Microscopic examination of the atherosclerotic aortae revealed prominent lesions with intimal thickening (Fig. 3). Despite similar elevations in tissue cholesterol content, intimal lesions had not yet developed in hypercholesterolemic vessels (Fig. 4). Intimal thickening was absent in control vessels. The endothelium appeared intact in all vessels on scanning and transmission electron microscopy studies.

Effects of cholesterol feeding on EDRF production. Detector vessels were preconstricted to a resting tension of $4.0 \pm 0.2$, $3.8 \pm 0.2$, and $4.0 \pm 0.2 \mathrm{~g}$ for hypercholesterolemic, atherosclerotic, and control vessel studies, respectively $(P=\mathrm{NS})$. Cholesterol feeding markedly impaired EDRF vasodilator activity as measured in bioassay (Fig. 5). Under basal flow conditions, effluent from normal vessels consistently caused relaxation of detectors. In contrast, effluent from both hypercholesterolemic and atherosclerotic vessels caused a tonic constriction. EDRF release elicited by the receptor-mediated agonist, acetylcholine $(1 \mu \mathrm{M})$, from normal vessels was sufficient to cause significant additional relaxations of detectors, but the vasodilator activity of EDRF released from hypercholesterolemic and atherosclerotic vessels was dramatically impaired. Effluent from normal vessels stimulated with A23187 $(10 \mu \mathrm{M})$ caused significant additional relaxations; and similar additional relaxations were evoked by EDRF produced from hypercholesterolemic vessels. However, atherosclerosis dramatically impaired the vasodilator activity of EDRF released by A23187. These conclusions were valid using an analysis either including or excluding the basal response (Fig. 5).

Detection of nitric oxide release by chemiluminescence. Despite significant impairment of the vasodilator activity of EDRF by cholesterol feeding, the quantity of nitric oxide recovered from the effluent of hypercholesterolemic and atherosclerotic vessels was significantly greater than in controls, and increased in response to agonists also (Fig. 6). The quantity of nitric oxide recovered from the effluent of hypercholesterolemic vessels was also greater than that from atherosclerotic vessels, although this did not reach statistical significance.

To contrast the vasodilator potencies of nitrogen oxides released from the aortae of normal, hypercholesterolemic, and atherosclerotic animals, the grams of relaxation produced by acetylcholine and the calcium ionophore is presented as a function of nitric oxide detected from each (Fig. 7). As is apparent, the amount of relaxation produced by the nitrogen oxides from hypercholesterolemic and atherosclerotic vessels is markedly reduced, suggesting that they have been released in a relatively inactive state.

Effects of L-NMMA on nitric oxide release and EDRF production. It has been suggested that multiple EDRFs exist (21). One explanation for impaired EDRF vasodilator activity in the presence of increased production of nitrosyl compounds is 


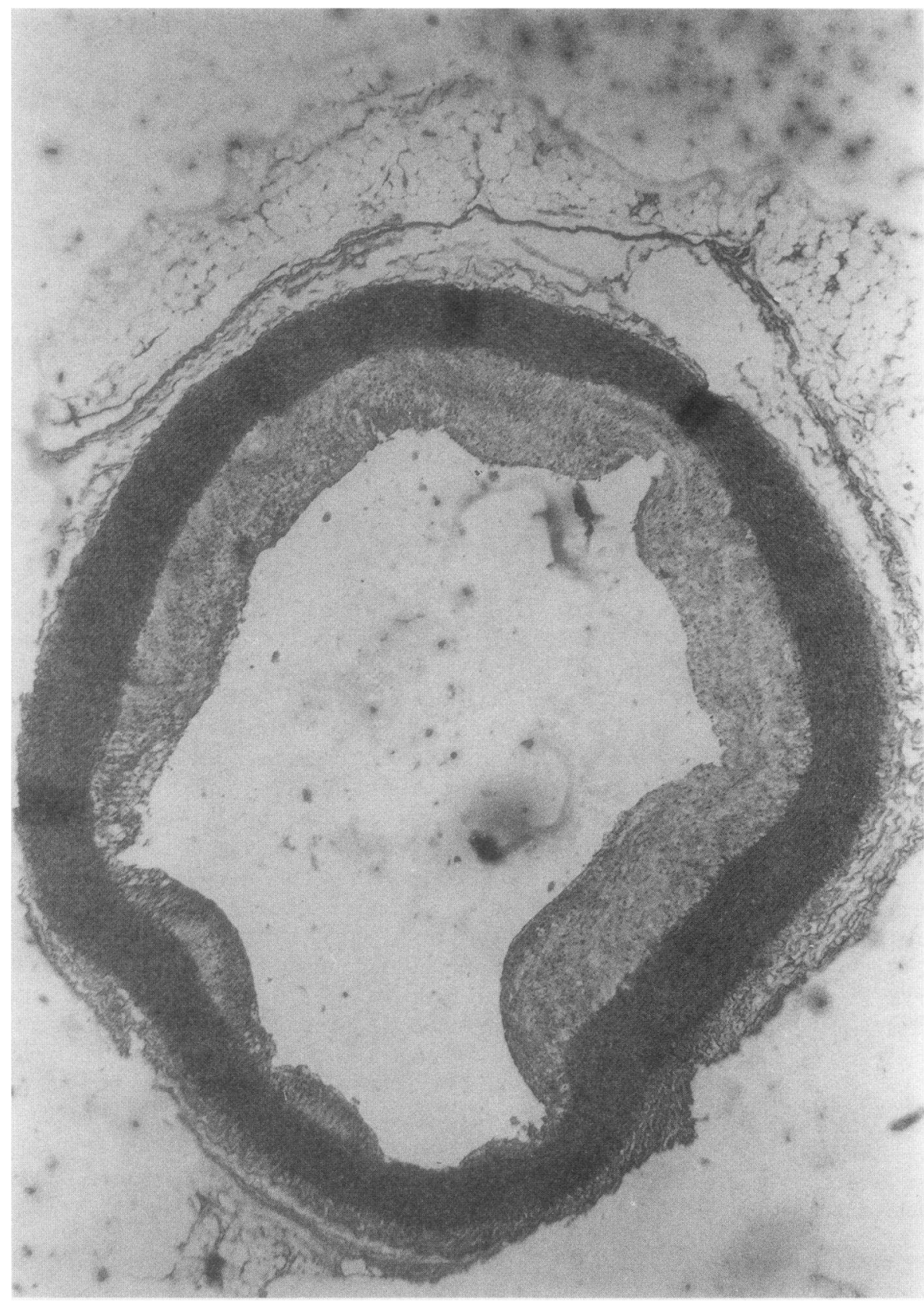

Figure 3. Histological cross-section (24X) of a representative atherosclerotic aorta demonstrating markedly thickened intima with prominent lesions. that cholesterol feeding impairs an EDRF unassociated with nitric oxide. To test this hypothesis, we examined the effect of a selective inhibitor of nitric oxide production, L-NMMA (5, $6)$, on EDRF released from normal rabbit aorta $(n=4)$. Nitric oxide release after L-NMMA $(300 \mu \mathrm{M})$ was undetectable during all study conditions. Relaxations to acetylcholine and A23187 were calculated from the formula: \% relaxation = (grams relaxation/grams of tension after basal response). The vasodilator activity of basally released EDRF, detected by bioassay, was abolished by L-NMMA and converted to a constriction of $21 \pm 8 \%$ (Fig. 8). L-NMMA had no direct effect on the denuded detector vessel. Acetylcholine $(1 \mu \mathrm{M})$ caused no detectable release of a relaxing substance, and the release of EDRF by A23187 (10 $\mu \mathrm{M})$ was nearly abolished (4 $\pm 3 \%$ relaxation). Thus, these experiments established that nitric oxide or a related nitrosyl compound is the primary EDRF produced by rabbit aorta, and that abnormalities of endothelium-dependent vasodilation induced by cholesterol feeding are solely related to a defect in the synthesis or release of this compound.

\section{Discussion}

The first important finding of this study was that both shortterm and long-term cholesterol feeding resulted in an apparent decrease in EDRF production, as evidenced by impaired vasodilator activity in bioassay. This finding confirms previous work (26). A second important finding was that the release of nitrosyl compounds from either hypercholesterolemic or atherosclerotic vessels was not reduced, but actually markedly increased. Finally, the production of these compounds from the vascular endothelium was further increased in response to acetylcholine and A23187 after cholesterol feeding. The incre- 

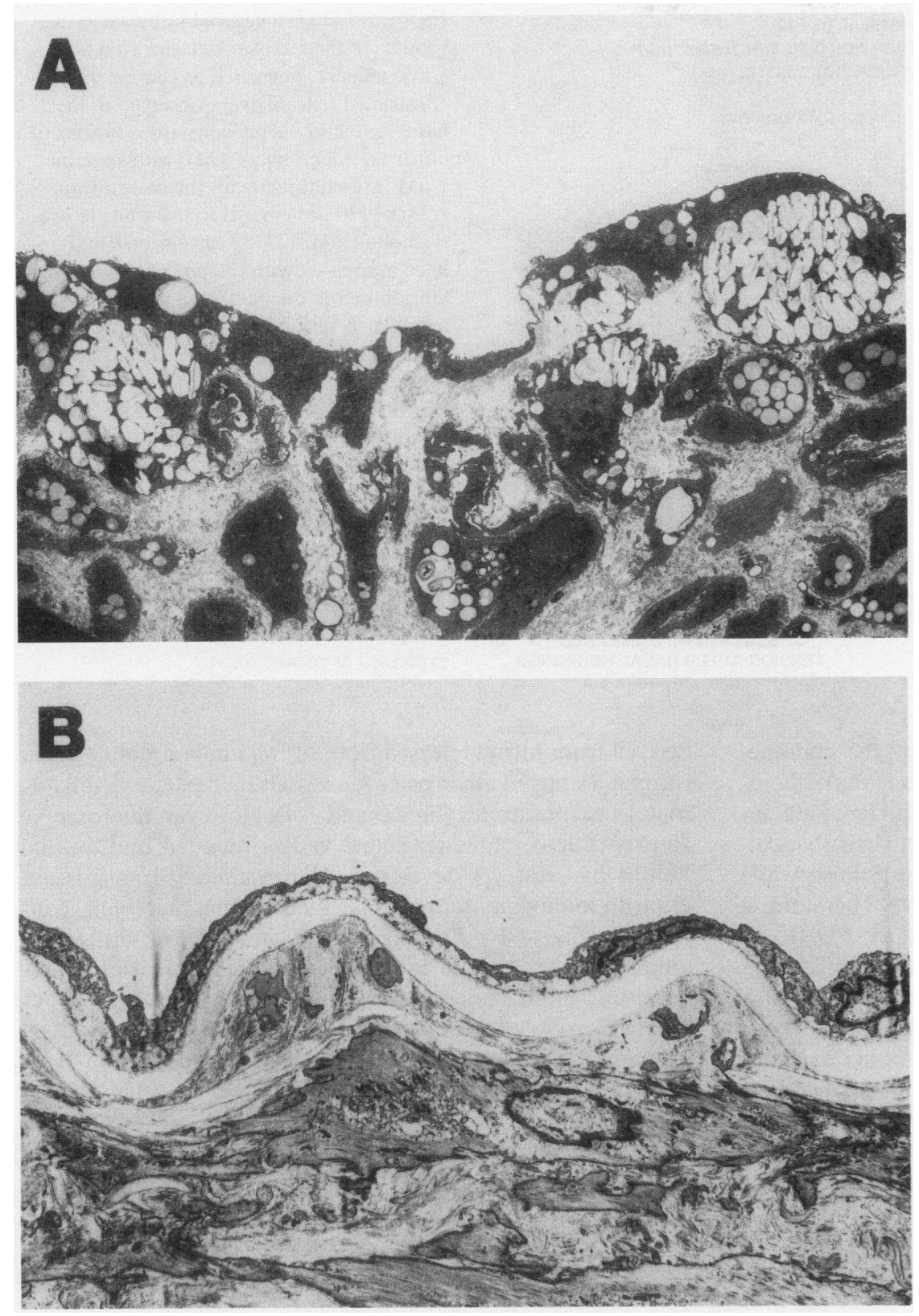

Figure 4. Transmission electron micrographs $(3,500 \times)$ comparing atherosclerotic $(A)$ and hypercholesterolemic $(B)$ vessels. Short-term cholesterol feeding did not result in intimal thickening or inflammatory cell infiltration. ment in nitric oxide synthesis after agonist administration was substantially greater than that observed from normal vessels under all conditions examined. These observations clearly demonstrate that the enzymatic process responsible for the production of nitric oxide by the vascular endothelium is not impaired by either short-term or long-term cholesterol feeding. Furthermore, neither hypercholesterolemia nor atherosclerosis impairs the signal transduction mechanisms responsible for activation of this enzyme.

The two most likely explanations for these observations are either that cholesterol feeding prevents incorporation of nitric oxide into a more potent nitrosylated compound, or that cholesterol feeding accelerates the intracellular or extracellular degradation of EDRF (either NO to $\mathrm{NO}_{2}^{-}$or R-NO to $\mathrm{R}^{-}$ $+\mathrm{NO}_{2}^{-}$). The methodology used to detect nitric oxide by chemiluminescence in this study and others $(1,3,4)$ does not distinguish free nitric oxide from a variety of nitrosylated compounds. We and others have found that one such compound, $S$-nitrosocysteine, more closely resembles EDRF than does nitric oxide $(4,29)$. It is possible that incorporation of nitric oxide into a compound such as a nitrosothiol may be prevented after cholesterol feeding. This could occur as a result of oxidation of endothelial cell sulfhydryl groups to the disulfide form. Thus, cholesterol feeding could deplete essential EDRF substrates and therefore prevent the release of nitrosylated compounds that are substantially more stable and potent than nitric oxide.

The increased release of oxygen radicals could also result in accelerated degradation of either nitric oxide or a related compound to an inactive form $(30,31)$. Products of oxidized LDL could contribute to this process (32).

There are several other less likely explanations for the increased release of apparently nonvasoactive nitrosyl compounds as a result of cholesterol feeding. 


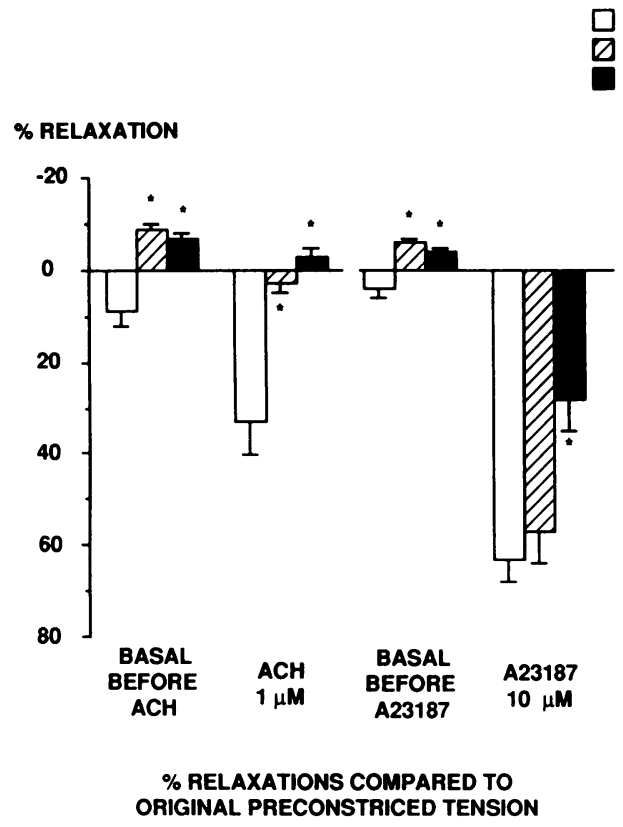

NORMAL $(n=10)$

HYPERCHOLESTEROLEMIC $(n=7)$

ATHEROSCLEROTIC $(n=8)$

$* p<0.05$ vs normal

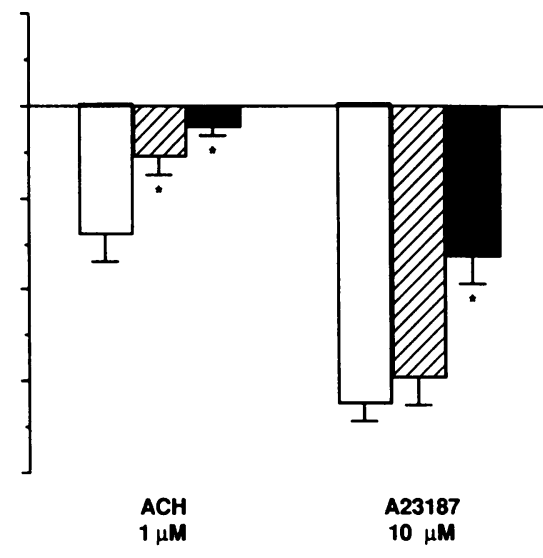

ORIGINAL PRECONSTRICED TENSION
\% RELAXATIONS COMPARED TO TENSION AFTER BASAL RESPONSE
Figure 5. EDRF measured in bioassay. Responses of the bioassay detector ring to aortic effluent, measured as changes in preconstricted tension, were observed in the basal state and during constant infusions of either acetylcholine $(1 \mu \mathrm{M})$ (with atropine $1 \mu \mathrm{M}$ infused directly on the detector), or A23187 $(10 \mu \mathrm{M})$. (Left) Relaxations to acetylcholine and A23187 have been calculated compared with the preconstricted tension before the basal response from the formula: \% relaxation $=($ [grams preconstricted tension before basal response - grams tension remaining after agonist]/ grams preconstricted tension before basal response). (Right) Relaxations to acetylcholine and A23187 have been calculated excluding the basal response from the formula: $\%$ relaxation $=$ (grams of relaxation/ grams of tension after the basal response). Negative values indicate constrictions. ${ }^{*} P$ $<0.05$ compared with control; ${ }^{+} P<0.05$ compared with hypercholesterolemia. Data expressed as mean \pm SEM.
If multiple relaxing factors are released by the endothelium, one explanation for the present findings is that atherosclerosis impairs an EDRF unrelated to nitric oxide. LNMMA, a competitive inhibitor of L-arginine, virtually abolished the release of any detectable relaxing substance while blocking the synthesis of nitric oxide (Fig. 8). Therefore, a nitrosylated compound appears to be the major EDRF released by rabbit aorta. Thus, it is unlikely that these findings could be explained by multiple relaxing factors.

Activated macrophages $(9,10,16)$ and neutrophils $(23)$ can produce nitric oxide and nitrite. Therefore, another explanation for our findings is that cholesterol feeding actually does impair nitric oxide synthesis from the endothelium, and that the excess nitric oxide detected by chemiluminescence was

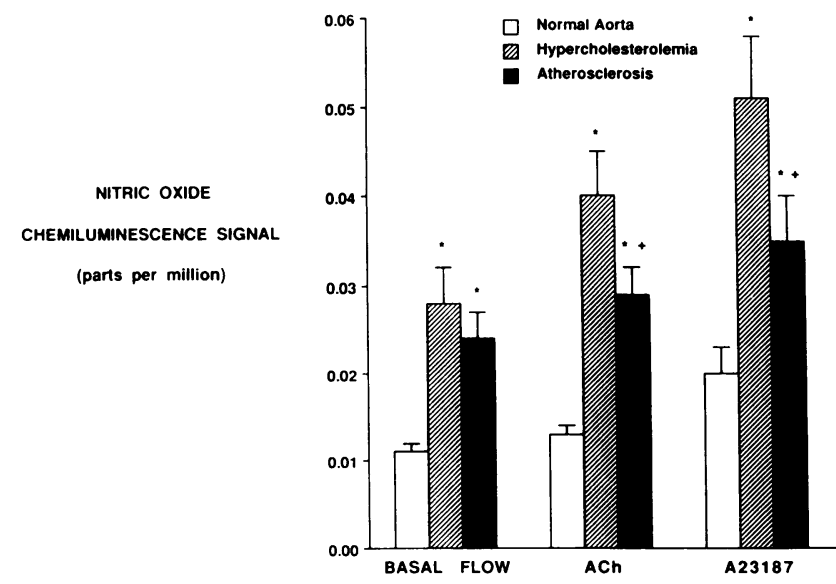

Figure 6. Nitric oxide measured by chemiluminescence. At the moment of maximal bioassay response, effluent from aortic segments was immediately diverted into an acid reflux chamber and nitric oxide content of the effluent was subsequently measured with a chemiluminescence technique. ${ }^{*} P<0.05$ compared with control; ${ }^{+} P$ $<0.05$ compared with hypercholesterolemia. Data expressed as mean \pm SEM. derived from nitrite released from intimal inflammatory cells. Intimal lesions in atherosclerotic vessels had extensive inflammatory cell infiltrates (Figs. 3 and $4 A$ ). However, microscopic studies of hypercholesterolemic vessels revealed that intimal lesions had not yet developed. Corresponding transmission electron micrographs also revealed no intimal macrophage infiltrates (Fig. $4 \mathrm{~B}$ ). Despite these histological findings, the amount of nitric oxide recovered from the effluent of hypercholesterolemic vessels was actually greater than that from atherosclerotic vessels. Therefore, the increased amount of nitric oxide detected after cholesterol feeding could not have been solely related to the presence of subendothelial macrophages.

A third explanation for our findings is that cholesterol feeding increases the release of vasoconstrictors from the endothelium. Constricting factors released in response to acetylcholine or the calcium ionophore could have reduced the apparent potency of EDRF released by these agonists, while not

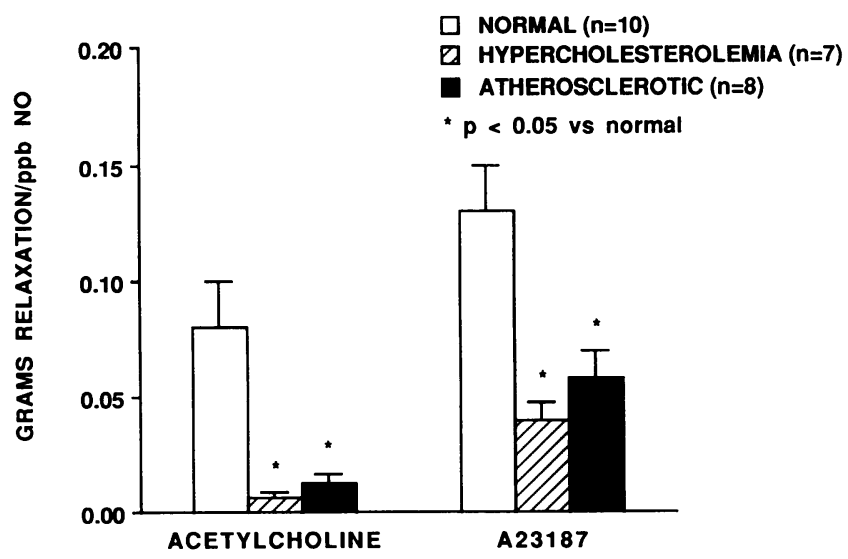

Figure 7. Grams relaxation produced by acetylcholine $(1 \mu \mathrm{M})$ or the calcium ionophore A23187 $(10 \mu \mathrm{M})$ expressed as a function of nitric oxide detected by chemiluminescence. 


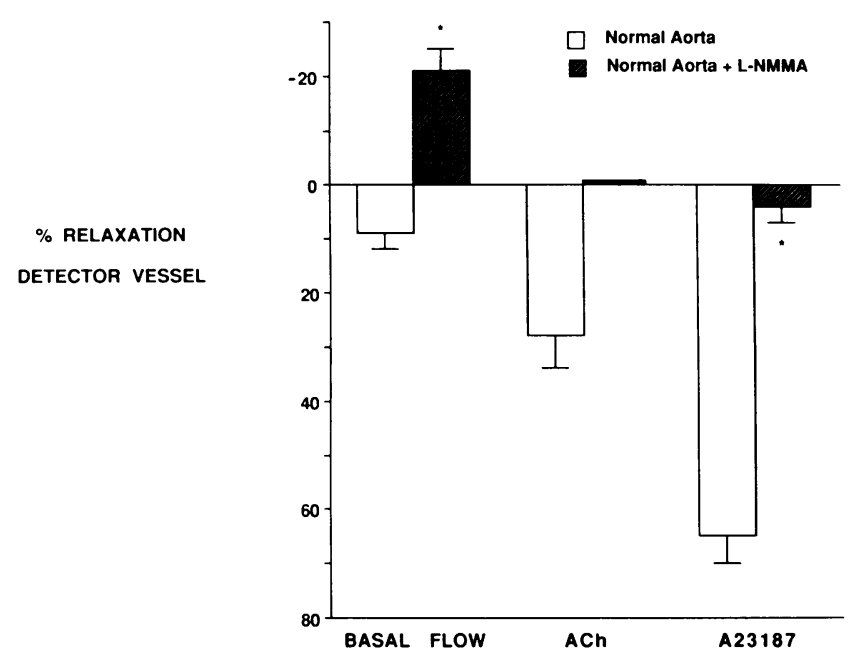

Figure 8. Effect of L-NMMA on EDRF release from normal rabbit aorta. L-NMMA completely abolished the release of nitrogen oxides. L-NMMA infusions also eliminated both basal and Ach stimulated release of EDRF. The release of EDRF evoked by A23187 was also virtually abolished. Data are expressed as the mean \pm SEM.

affecting nitric oxide signals. In previous studies we have been unable to detect the excess release of vasoconstrictor factors from the aortae of cholesterol-fed rabbits. In this study, the aortae of cholesterol-fed rabbits released a constricting factor under basal conditions. This, however, likely represented the effect of normally released constrictor factors by the endothelium, as L-NMMA also unmasked a similar basal constriction from normal vessels (Fig. 7). The known constricting factors include the polypeptide endothelin (34) and one or more products of the cyclooxygenase pathway $(35,36)$. It is not known if cholesterol feeding alters the production of endothelin. Enhanced release of prostanoids could not have accounted for our observations because experiments were performed in the presence of indomethacin.

In these studies, the production of nitrosyl compounds with minimal vasodilator activity by hypercholesterolemic and atherosclerotic vessels was increased compared with normal vessels. Further augmentation of release of these compounds in response to agonists clearly shows that the signal transduction mechanisms responsible for nitric oxide synthesis are not impaired as a result of atherosclerosis. This finding is in clear contradistinction to conclusions drawn from observations of endothelium-dependent vascular relaxation of intact atherosclerotic vessel rings (24). Further, these studies illustrate the importance of directly assessing the activity of the enzyme responsible for production of nitrosyl compounds (and presumably EDRF), rather than indirect assessment of EDRF release via simple measurements of endothelium-dependent relaxations in intact vascular rings. Arginine deficiency also does not appear to result from cholesterol feeding. These findings strongly suggest that the enzymatic process responsible for nitric oxide synthesis is upregulated, rather than impaired, as a result of cholesterol feeding. It is interesting to speculate that either the inability to synthesize a parent nitrosylated compound, or the accelerated intracellular degradation of EDRF, leads to a loss of feedback inhibition increasing the production of nitrosyl compounds from arginine. Thus, an important autocrine function for EDRF may exist.
Conclusions. Cholesterol feeding results in upregulation of the synthesis of nitric oxide or related nitrosyl compounds from the vascular endothelium while dramatically impairing the vasodilator activity of EDRF. These observations cannot be explained by the impairment of an EDRF unrelated to nitric oxide, by excess release of nitric oxide and nitrite by inflammatory cells, or by the enhanced release of constricting factors by the endothelium. Impaired endothelium-dependent vasodilation may be due to prevention of nitric oxide incorporation into a more potent nitrosylated compound, perhaps by depletion of essential EDRF substrate. Alternatively, accelerated intracellular or extracellular degradation of EDRF may occur. Finally, our observations do not support an impairment in signal transduction within the vascular endothelium, and exclude deficiency of arginine in hypercholesterolemic and atherosclerotic endothelium as a cause of abnormal endothelium-dependent vascular relaxations.

\section{Acknowledgments}

We are grateful to Pam Tompkins for the preparation of histological sections, Tim Hofmeyer for technical assistance, and Marlene Blakley for preparing the manuscript.

Dr. Minor is the recipient of an National Heart, Lung and Blood Institute Research Fellowship Award. Dr. Myers is a Research Associate of the Veterans Administration. Dr. Guerra is a recipient of a Stanley J. Sarnoff Cardiovascular Research Fellowship. Dr. Harrison is an Established Investigator of the American Heart Association. Supported by National Institutes of Health grant HL-32717, U.S. Public Health Service grant HL-0712114, Atherosclerosis Specialized Center of Research HL-14230, and a Veterans Administration Merit Award.

\section{References}

1. Palmer, R. M. J., A. G. Ferrige, and S. Moncada. 1987. Nitric oxide release accounts for the biological activity of endothelium-derived relaxing factor. Nature (Lond.). 327:524-526.

2. Ignarro, L. J. 1989. Biological properties of endothelium-derived nitric oxide formed and released from artery and vein. Circ. Res. 65:1-21.

3. Myers, P. R., R. Guerra, and D. G. Harrison. 1989. Release of NO and EDRF from cultured bovine aortic endothelial cells. Am. J. Physiol. 256(Heart Circ. Physiol. 25):H1030-H1037.

4. Myers, P. R., R. L. Minor, R. Guerra, Jr., J. N. Bates, and D. G. Harrison. 1990. The vasorelaxant properties of the endothelium-derived relaxing factor more closely resemble S-nitrosocysteine than nitric oxide. Nature (Lond.). 345:161-163.

5. Schmidt, H. H. H. W., P. Wilke, B. Ever, and E. Bohme. 1989. Enzymatic formation of nitrogen oxides from L-arginine in bovine brain cytosol. Biochem. Biophys. Res. Commun. 165:284-291.

6. Garthwaite, J., E. Southam, and M. Anderton. 1989. A kainate receptor linked to nitric oxide synthesis from arginine. J. Neurochem. 53:1952-1954.

7. Curran, R. D., T. R. Billiar, D. J. Stuehr, K. Hofmann, and R. L. Simmons. 1989. Hepatocytes produce nitrogen oxides from L-arginine in response to inflammatory products of Kupffer cells. J. Exp. Med. 170:1769-1774.

8. Mayer, B., and E. Boehm. 1989. $\mathrm{Ca}^{2+}$-dependent formation of an L-arginine-derived activator of soluble guanylyl cyclase in bovine lung. FEBS (Fed. Eur. Biol. Soc.) Lett. 256:211-214.

9. Hibbs, J. B., R. R. Taintor, and Z. Vavrin. 1987. Macrophage cytotoxicity: role for L-arginine deiminase and imino nitrogen oxidation to nitrite. Science (Wash. DC). 235:473-476.

10. Marletta, M. A., P. S. Yoon, R. Iyengar, C. D. Leaf, and J. S. Wishnok. 1988. Macrophage oxidation of $\mathrm{L}$-arginine to nitrite and nitrate: nitric oxide is an intermediate. Biochemistry. 24:8706-8711. 
11. Palmer, R. M. J., D. S. Ashton, and S. Moncada. 1988. Vascular endothelial cells synthesize nitric oxide from L-arginine. Nature (Lond.). 333:664-666.

12. Palmer, R. M. J., D. D. Rees, D. S. Ashton, and S. Moncada. 1988. L-arginine is the physiological precursor for the formation of nitric oxide in endothelium-dependent relaxation. Biochem. Biophys. Res. Commun. 153:1251-1256.

13. Förstermann, U., R. Z. Huang, and F. Murad. 1989. Subcellular localization of the enzyme(s) responsible for the synthesis of the endothelium-derived relaxing factor (EDRF) in endothelial cells. Clin. Res. 37:877a. (Abstr.)

14. Mayer, B., K. Schmidt, P. Humbert, and E. Bohme. 1989. Biosynthesis of endothelium-derived relaxing factor: a cytosolic enzyme in porcine aortic endothelial cells $\mathrm{Ca}^{2+}$-dependently converts L-arginine into an activator of soluble guanylyl cyclase. Biochem. Biophys. Res. Commun. 164:678-685.

15. Bredt, D. S., and S. H. Snyder. 1990. Isolation of nitric oxide synthetase, a calmodulin-requiring enzyme (endothelium-derived relaxing factor/arginine/cGHM). Proc. Natl. Acad. Sci. USA. 87:682685.

16. Tayeh, M., and M. A. Marletta. 1989. Macrophage oxidation of L-arginine to nitric oxide, nitrite, and nitrate. Tetrahydrobioterin is required as a cofactor. J. Biol. Chem. 264:19654-19658.

17. Jayakody, R. L., M. P. J. Senaratne, A. B. R. Thomson, and C. T. Kappagoda. 1985. Cholesterol feeding impairs endothelial-dependent relaxation of rabbit aorta. Can. J. Physiol. Pharmacol. 63:1206-1209.

18. Freiman, P. C., G. C. Mitchell, D. D. Heistad, M. L. Armstrong, and D. G. Harrison. 1986. Atherosclerosis impairs endothelial-dependent vascular relaxation to acetylcholine and thrombin in primates. Circ. Res. 58:783-789.

19. Sreeharan, N., R. L. Hayakody, M. P. J. Senaratne, A. B. R. Thomson, and C. T. Kappagoda. 1986. Endothelial-dependent relaxation and experimental atherosclerosis in the rabbit aorta. Can. J. Physiol. Pharmacol. 64:1451-1453.

20. Verbeuren, T. J., F. H. Jordaens, L. L. Zonnekeyn, H. CeVan, M. C. Coene, and A. G. Herman. 1986. Effect of hypercholesterolemia on vascular reactivity in the rabbit. I. Endothelial-dependent and endothelial-independent contractions and relaxation in isolated arteries of control and hypercholesterolemic rabbits. Circ. Res. 58:552-564.

21. Chappell, S. P., M. J. Lewis, and A. H. Henderson. 1987. Effect of lipid feeding on endothelial-dependent vascular relaxation in rabbit aorta preparation. Cardiovasc. Res. 21:34-38.

22. Harrison, D. G., P. C. Freiman, M. L. Armstrong, M. L. Marcus, and D. D. Heistad. 1987. Alterations of vascular reactivity in atherosclerosis. Circ. Res. 61:II-74-II-80.

23. Harrison, D. G., M. L. Armstrong, P. C. Freiman, and D. D. Heistad. 1987. Restoration of endothelial-dependent relaxation by dietary treatment of atherosclerosis. J. Clin. Invest. 80:1808-1811.
24. Bossaler, C., G. B. Habib, H. Yamomoto, C. Williams, S. Wells, and P. D. Henry. 1987. Impaired muscarinic endothelial-dependent relaxation and cyclic guanosine 5-monophosphate formation in atherosclerotic human coronary artery and rabbit aorta. J. Clin. Invest. 79:170-174.

25. Forstermann, U., A. Mügge, U. Acheid, A. Haverich, and J. C. Frolich. 1988. Selective attenuation of endothelial-mediated vasodilation atherosclerotic human coronary arteries. Circ. Res. 62:185-190.

26. Guerra, R., Jr., A. F. A. Brotherton, P. J. Goodwin, C. R. Clark, M. L. Armstrong, and D. G. Harrison. 1989. Mechanisms of abnormal endothelium-dependent responses in atherosclerosis. Blood Vessels. 26:300-314

27. Lipid Research Clinics Program. Manual and Laboratory Operations. 1974. Lipid and Lipoprotein Analysis. Vol. 1. DHEW Publication No. (NIH) 75-628. US Government Printing Office, Washington, DC

28. Rubanyi, G. M., and P. M. Vanhoutte. 1987. Nature of endothelium-derived relaxing factor: are there two relaxing mediators? Circ Res. 61(Suppl II):II-61-II-67.

29. Rubanyi, G. M., A. Johns, D. G. Harrison, and D. Wilcox. 1989. Evidence that EDRF may be identical with an S-nitrosothiol and not with free nitric oxide. Circulation. 80(Suppl II):II-281.

30. Rubanyi, G. M., and P. M. Vanhoutte. 1986. Superoxide anions and hyperoxia inactivate endothelium-derived relaxing factor. Am. J. Physiol. 250:H822-H827.

31. Gryglewski, R. J., R. M. J. Palmer, and S. Moncada. 1986. Superoxide anion in involved in the breakdown of endothelium-derived vascular relaxing factor. Nature (Lond.). 320:454-456.

32. Kugiyama, K., M. Bucay, J. D. Morrisett, R. Roberts, and P. D. Henry 1989. Oxidized LDL impairs endothelium-dependent arterial relaxation. Circulation. 80(Suppl II):II-279.

33. Rimele, T. J., R. J. Sturm, L. M. Adams, D. E. Henry, R. J. Heaslip, B. M. Weichman, and D. Grimes. 1988. Interaction of neutrophils with vascular smooth muscle: identification of a neutrophilderived relaxing factor. J. Pharm. Exp. Ther. 245:102-111.

34. Yanagisawa, M., H. Kurihara, S. Kimura, Y. Tomobe, M. Kabayashi, Y. Mitsui, Y. Yazaki, K. Goto, and T. Masaki. 1988. A novel potent vasoconstrictor peptide produced by vascular endothelial cells. Nature (Lond.). 332:411-415.

35. Shirahase, H., H. Usui, K. Kurahashi, and M. Fujiwara. 1987. Possible role of endothelial thromboxane $A_{2}$ in the resting tone and contractile responses to acetylcholine and arachidonic acid in canine cerebral arteries. Cardiovasc. Pharmacol. 10:517-522.

36. Katusic, Z. S., and P. M. Vanhoutte. 1986. Anoxic contractions in isolated canine cerebral arteries. Contribution of endothelium-derived factors, metabolites or arachidonic acid and calcium entry. $J$. Cardiovasc. Pharmacol. 8:S597-S101. 